EXPERIMENTAL STUDY

\title{
Experimentally-induced hyperthyroidism is associated with activation of the rat hypothalamic-pituitary-adrenal axis
}

\author{
Elizabeth O Johnson ${ }^{1,2,3}$, Themis C Kamilaris ${ }^{1,2}$, Aldo E Calogero ${ }^{1}$, Philip W Gold ${ }^{2}$ and George P Chrousos ${ }^{1,4}$ \\ ${ }^{1}$ Department of Anatomy-Histology-Embryology, University of Ioannina, School of Medicine, Ioannina 45110, Greece, ${ }^{2}$ Developmental Endocrinology \\ Branch, National Institute of Child Health and Human Development and ${ }^{3}$ Neuroendocrinology Branch, National Institute of Mental Health, Bethesda, \\ Maryland 20892, USA and ${ }^{4}$ Department of Pediatrics, University of Athens, School of Medicine, Athens, Greece
}

(Correspondence should be addressed to E O Johnson, Department of Anatomy-Histology-Embryology, University of Ioannina, School of Medicine, Ioannina 45110,Greece; Email: ejohnson@cc.uoi.gr or soukakos@panafonet.gr)

Elizabeth O Johnson and Themis C Kamilaris contributed equally to this project

\begin{abstract}
Objective: Previous studies on the effects of altered thyroid function on the secretion and metabolism of adrenocortical hormones suggest a degree of adrenocortical hyperactivity in hyperthyroidism. We have previously shown that experimentally-induced hyperthyroidism is associated with significant alterations in pituitary-adrenal responsiveness to synthetic ovine corticotropin-releasing hormone (oCRH) that are contingent upon the duration of the altered thyroid function. The purpose of this study was to assess the time-dependent effects of hyperthyroidism on the functional integrity of the hypothalamic-pituitary-adrenal (HPA) axis by in vivo stimulation of the hypothalamic $\mathrm{CRH}$ neuron and adrenal cortex.

Methods: The functional integrity of the HPA axis was examined in vivo in sham-thyroidectomized male Sprague-Dawley rats given placebo or in thyroidectomized rats given $50 \mu \mathrm{g}$ of thyroxine every day for 7 or 60 days. Responses to insulin-induced hypoglycemia and IL-1 $\alpha$ stimulation were used to assess the hypothalamic $\mathrm{CRH}$ neuron. Adrenocortical reserve was assessed in response to low-dose adrenocorticotropic hormone (ACTH), following suppression of the HPA axis with dexamethasone. Adrenal and thymus tissue weight, in addition to basal plasma ACTH, corticosterone and thyroid indices were also determined.

Results: Basal plasma corticosterone and corticosterone binding globulin (CBG) concentrations were significantly increased in short- and long-term hyperthyroid rats, and by 60 days, cerebrospinal fluid (CSF) corticosterone levels were significantly increased. Basal plasma ACTH levels were similar to controls. Although plasma ACTH responses to hypoglycemic stress and IL- $1 \alpha$ administration in both short- and long-term hyperthyroidism were normal, corticosterone responses to the ACTH release during the administration of these stimuli were significantly increased. The adrenal reserve was significantly elevated in short-term hyperthyroidsim. Long-term hyperthyroidism, however, was associated with a significant reduction in adrenocortical reserve. A significant increase in adrenal weights and a decrease in thymus weights were observed in both short- and long-term hyperthyroidism. Conclusions: The available data confirms that hyperthyroidism is associated with hypercorticosteronemia, although the locus that is principally affected still remains unclear. Despite the sustained hyperactivity of the HPA axis, long-term experimentally-induced hyperthyroidism is associated with diminished adrenal functional reserve. The alterations in HPA function in states of disturbed thyroid function were found to be somewhat more pronounced as the duration of thyroid dysfunction increased.
\end{abstract}

European Journal of Endocrinology 153 177-185

\section{Introduction}

Thyrotoxicosis in humans and experimental animals is thought to be associated with hyperactivity of the hypothalamic-pituitary-adrenal (HPA) axis (1-6). This conclusion is based on measurement of the concentrations of total plasma corticosteroids $(7,8)$, excretion of urinary 17-hydroxysteroids, and assessment of the adrenocortical response to the synthetic adrenocorticotropic hormone $(\mathrm{ACTH})_{1-24}(9-11)$. None of these measures, however, definitively establishes that thyrotoxicosis is associated with true hyperfunction of the HPA axis. Moreover, these studies do not identify the specific site of the HPA axis that is most affected or whether the duration of hyperthyroidism influences the functional activity of the HPA axis. 
We have previously reported on the impact of alterations in thyroid function on pituitary-adrenal responses to corticotropin-releasing hormone (CRH). Our results showed, as early as 7 days after experimentally-induced hyperthyroidism, a slight attenuation in the ACTH response to ovine (o)CRH, in association with a significant augmentation of the corticosterone response to the ACTH released during CRH stimulation (2). These data were found most compatible with a subtle hyperthyroidism-induced centrally mediated hyperstimulation of the adrenals in rats with short- and longterm hyperthyroidism.

Previous studies have extensively examined the effects of altered thyroid function on the secretion and metabolism of adrenocortical hormones. Thus, in thyrotoxic states the degradation of cortisol is accelerated, but its rate of production is also increased, so that circulating levels of cortisol remain normal $(5,10,12$, 13). These observations indicate that a degree of adrenocortical hyperactivity would be sustained in hyperthyroidism as a response to increased need. Apart from data indicating accelerated disposal of cortisol in hyperthyroidism and increased number of cortisol secretory episodes $(6,14)$, there are few studies regarding the impact of hyperthyroidism on adrenal functional reserve, most of which are conflicting.

The present study explores the impact of hyperthyroidism on the overall organization and functional activity of the HPA axis. We report the effects of experimentally induced hyperthyroidism on the in vivo responsiveness of the hypothalamic $\mathrm{CRH}$ neuron by assessing the plasma immunoreactive (IR) ACTH and IR-corticosterone responses to insulin-induced hypoglycemia and IL- $1 \alpha$ stimulation. To assess the impact of hyperthyroidism on adrenocortical reserve, we measured the corticosterone responses to stimulation with low-dose ACTH, after dexamethasone suppression. To determine the potential effects of the duration of altered thyroid status on HPA axis function, we conducted studies at 7 and 60 days after experimentallyinduced hyperthyroidism. In addition, as estimates of the impact of hyperthyroidism on the overall functional activity of the HPA axis, we measured the cerebrospinal fluid (CSF) corticosterone concentrations, as an estimate of plasma free corticosterone concentrations, plasma corticosteroid-binding globulin (CBG) and followed changes in adrenal and thymus weight.

\section{Materials and methods}

\section{In vivo studies}

Experiments were performed on male Sprague-Dawley rats weighing 350-400 g (Charles River, Wilmington, MA, USA) and housed for 1-2 weeks under an artificial 12 light: $12 \mathrm{~h}$ dark cycle with lights on from $0600 \mathrm{~h}$. The room was kept at $24{ }^{\circ} \mathrm{C}$ with controlled humidity. Rat chow (Ralston-Purina, St Louis, MO, USA) and water were available ad libitum. Surgical thyroidectomy (Tx) or sham Tx was carried out under methoxyflurane inhalation anesthesia (Metofane, Pitman-Moore, Washington Grossing, NJ, USA). After recovery, animals were supplied with $1 \%$ calcium lactate solution as drinking water to avoid hypocalcemia from possible excision of the parathyroid glands. Experiments were performed 7 and 60 days after Tx or sham Tx and daily injections of s.c. thyroxine (T4; $50 \mathrm{mg}$ ) (Sigma) or placebo treatment, respectively. Euthyroid and hyperthyroid animals were defined by sham $\mathrm{Tx}+$ placebo and $\mathrm{Tx}+\mathrm{T} 4$ treatments, respectively. All procedures were approved by the NIH Committee for the use and welfare of laboratory animals and conformed to the International Ethical Standards (86/609-EEC) for the care and use of laboratory animals.

Twenty-four hours before the acute experiments, the animals were implanted with indwelling i.v. cannulae as previously described (2). Briefly, cannulae were prepared from a $35 \mathrm{~cm}$ length of polyethylene tubing $(0.965 \mathrm{~mm}$, od; $0.58 \mathrm{~mm}$ id; PE-50, Clay Adams, Pasippany, NJ, USA). A $3 \mathrm{~cm}$ length of silastic tubing (1.85 mm outside diameter (od); $0.98 \mathrm{~mm}$ inside diameter (id); Dow-Corning, Midland, MI, USA) was passed $0.4 \mathrm{~cm}$ over one end of the polyethylene tubing. The silastic end of the catheter was inserted under light methoxyflurane inhalation anesthesia into the jugular vein and advanced into the right atrium. The cannula was run under the skin of the back, externalized at the nape of the neck, and sheathed in a stainless steel spring coil (Alice King Chatman, Los Angeles, CA, USA) that was anchored to the nape of the neck by a suture. The cannula extended from the coil approximately $5 \mathrm{~cm}$. Cannulae were flushed with $0.3 \mathrm{ml}$ of sterile saline solution containing $20 \mathrm{U} / \mathrm{ml}$ heparin (Elkins-Sinn, Cherry Hill, NJ, USA). After cannulation, the animals were allowed to awaken from anesthesia and were caged individually in plexiglass cages where they could move freely. The rats were shielded from seeing movement outside the cages and were kept in a quiet room during the stimulation tests.

\section{Basal hormonal levels}

Basal plasma ACTH, corticosterone and thyroid indices were determined at the time of killing. Basal plasma corticosteroid binding globulin (CBG) binding capacity was measured in euthyroid and hyperthyroid animals as previously described (2). Briefly, endogenous corticosterone was stripped and plasma was diluted 1:20 with $50 \mathrm{mM}$ PBS. Diluted plasma was incubated with [3H]-corticosterone for $90 \mathrm{~min}$ at $4{ }^{\circ} \mathrm{C}$. Nonspecific binding was determined for each sample by adding non-radioactive corticosterone to a duplicate sample and incubating under the same conditions. The bound and unbound $[3 \mathrm{H}]$-corticosterone was separated with dextran-coated charcoal. CBG binding capacity was defined as total binding minus the nonspecific binding corrected to micrograms [3H]-corticosterone bound/dl rat plasma. Levels 
of IR-ACTH and IR-corticosterone were assessed in CSF as we have previously described (2). Briefly, rats were anesthetized [pentobarbital $(55 \mathrm{mg} / \mathrm{kg}$ body weight (BW), i.p.) and ketamine (50 mg/kg BW, i.p.)] and placed in a stereotaxic apparatus. CSF samples $(100-200 \mu \mathrm{l})$ were drawn from the cisterna magna before and after thyroidectomy plus treatment (placebo or T4).

\section{Hypothalamic CRH neuron - insulin-induced hypoglycemia and $I L-1 \alpha$ stimulation}

The day after the cannulation, regular insulin $(3.0 \mathrm{U} / \mathrm{kg}$ BW) or vehicle was injected as an i.v. bolus (over approximately $30 \mathrm{~s}$ ) through the cannulae between 1200 and $1500 \mathrm{~h}$. Blood samples $(0.3 \mathrm{ml})$ for plasma ACTH and corticosterone, as well as for serum glucose determinations were drawn from the cannulae immediately before and 15, 30 and $60 \mathrm{~min}$ after the insulin or vehicle was administered. The blood volume was maintained by the injection of an equal volume heparinized saline immediately following the blood withdrawal.

IL- $1 \alpha$ stimulation test was performed in overnight fasted rats, 60 days after thyroidectomy plus T4 treatment. The day after cannulation, recombinant IL- $1 \alpha$ (Hoffman-Lacrose) $10 \mu \mathrm{g} / \mathrm{kg}$ BW or vehicle was injected in a $0.3 \mathrm{ml}$ volume of $0.04 \mathrm{M}$ phosphate buffer, pH 7.4 as an i.v. bolus through the cannulae. There were five or six rats per treatment group. Blood samples $(0.3 \mathrm{ml})$ for plasma ACTH and corticosterone determination were drawn from the cannulae immediately before and 5, 15, 30, 60, 90 and $120 \mathrm{~min}$ after the IL- $1 \alpha$ or vehicle were administered. The blood volume was maintained with physiological saline.

\section{Adrenal cortex - ACTH stimulation}

On the day of the experiment and $3 \mathrm{~h}$ before the stimulation test, all animals were injected i.m. with $200 \mu \mathrm{g}$ dexamethasone to inhibit endogenous ACTH secretion. Synthetic $\mathrm{ACTH}_{1-24}(25 \mu \mathrm{g} / \mathrm{kg} \mathrm{BW})$ was given in i.v. bolus through the cannulae in both eu- and hyperthyroid groups at 7 and 60 days after treatment. Blood samples for corticosterone determinations were drawn from the cannulae immediately before and 15, 30, 60 and $90 \mathrm{~min}$ after $\mathrm{ACTH}_{1-24}$ or vehicle administration.

In all in vivo experiments blood samples were collected in tubes containing EDTA $(20 \mu \mathrm{g} / \mathrm{ml})$ chilled immediately in an ice bath, and processed as previously described (15). Plasma was separated and stored at $-20{ }^{\circ} \mathrm{C}$ until the assays were run. Each rat was used in only one experiment.

\section{Thymus and adrenal weights}

Adrenals and thymuses were dissected out of animals killed by decapitation. The right adrenal gland was defatted and its wet weight measured immediately after removal from the rat. The thymus was cleaned, excess tissue was dissected away, patted dry and immediately weighed.

\section{Hormone and other determinations}

ACTH was measured by RIA in extracted plasma. ACTH was assayed as previously described (16) using an anti-ACTH serum (IgG Corp., Nashville, TN, USA) which is directed at the ACTH- $(5-18)$ sequence. The intraassay coefficient of variation $(\mathrm{CV})$ for the ACTH RIA was $4.6 \%$ and the corresponding interassay CV was $8.6 \%$ at $60 \mathrm{pg} / \mathrm{ml}$. Sensitivity was $5 \mathrm{pg} / \mathrm{ml}$. Immunoreactive (IR)-corticosterone was measured directly in unextracted plasma by RIA using a [125I]corticosterone kit from Radioassay System Laboratory, Inc. (Carson, CA, USA). Intraassay CV was $1.8 \%$ and interassay CVs were $1.6 \%$ at $80 \mathrm{ng} / \mathrm{ml}$ and $1.9 \%$ at $475 \mathrm{ng} / \mathrm{ml}$. Sensitivity was $4.5 \mathrm{ng} / \mathrm{ml}$.

Plasma TSH concentrations were determined by RIA using an anti-rat TSH serum and purified rat TSH reference preparation provided by the National Hormone and Pituitary Program (Baltimore, MD, USA). Plasma total T4 (TT4) and total T3 (TT3) concentrations were determined by RIA (Immunochem Corp., Carson CA, USA).

\section{Statistical analyses}

Results are expressed as the means \pm S.E.M. throughout the study. The effects of insulin, IL- $1 \alpha$ and ACTH on ACTH and/or corticosterone release were analyzed using two-way analysis of variance (ANOVA), followed by the Fisher's PLSD test. The total area under the curve (AUC) of ACTH and corticosterone, after stimulation, was calculated by integration of hormone levels in conventional units and time of testing in minutes. The AUC net from the baseline (net AUC) was calculated as the difference between total AUC and basal AUC (baseline times length of the testing). Delta peaks were calculated as the difference between the peak response and baseline (mean of the two basal determinations). Analyses of peak, delta, total and net AUC of plasma ACTH and corticosterone responses were performed using one-way ANOVA followed by Fisher's PLSD. Hormone values below the detection limit of the assay were assigned a value half that of the limit of detection.

\section{Results}

\section{In vivo studies - basal hormonal levels}

The mean basal TT4 levels were significantly increased in rats given exogenous thyroid hormone administration at both 7 and 60 days compared with euthyroid rats $(11.8 \pm 1.0$ and $7.6 \pm 0.6 \mu \mathrm{g} / \mathrm{ml}$ vs $3.9 \pm 0.2$ and $3.5 \pm 0.2 \mathrm{ug} / \mathrm{ml}$ at 7 and 60 days respectively; $P<0.0001$, by ANOVA). TT3 levels were also significantly elevated in hyperthyroid rats (121.8 \pm 7.9 
and $119.3 \pm 15.3 \mathrm{ng} / \mathrm{ml}$ at 7 and 60 days respectively) compared with euthyroid animals $(39.0 \pm 3.1$ and $39.5 \pm 2.9$ at 7 and 60 days respectively). This was associated with a significant decrease in the concurrent mean plasma thyroid-stimulating hormone (TSH) concentrations $(0.8 \pm 0.06$ and $0.7 \pm 0.06 \mathrm{ng} / \mathrm{ml}$ at 7 and 60 days respectively) compared with values in euthyroid rats $(4.2 \pm 0.4$ and $2.8 \pm 0.4 \mathrm{ng} / \mathrm{ml}$ at 7 and 60 days respectively; $P<0.0001$, by ANOVA).

Short-term hyperthyroid rats tended to have higher plasma ACTH levels than euthyroid controls $(123 \pm 6.2$ and $90.3 \pm 4.6 \mathrm{pg} / \mathrm{ml} ; P=0.06$ ), while there was no difference between long-term hyperthyroid rats and controls $(118.1 \pm 11.3$ and $95.8 \pm 6.9 \mathrm{pg} / \mathrm{ml})$. Mean basal plasma corticosterone levels were significantly increased in both short- and long-term hyperthyroid rats compared with euthyroid ( $240.6 \pm 36.4$ and $422.6 \pm 33.4 \mathrm{ng} / \mathrm{ml}$ vs $137.2 \pm 25.5$ and $99.6 \pm 21.0 \mathrm{ng} / \mathrm{ml}$ at 7 and 60 days, respectively; $P<0.05$, ANOVA followed by Fisher PLSD). Basal plasma CBG binding capacity was also significantly elevated in short- and long-term hyperthyroid animals compared with control (27.9 \pm 3.3 and $29.8 \pm 2.0 \mu \mathrm{g} / \mathrm{dl} \quad$ vs $14.1 \pm 0.7$ vs $13.2 \pm 0.6 \mu \mathrm{g} / \mathrm{dl}$ respectively; $P \leq 0.05$, ANOVA followed by Fisher PLSD). CSF IR-corticosterone levels were significantly increased at 60 days of hyperthyroidism compared with euthyroid levels $(P \leq 0.05$, ANOVA followed by Fisher PLSD).

\section{Hypothalamic CRH neuron - responses to insulin-induced hypoglycemia and $I L-1 \alpha$ stimulation}

The plasma ACTH response to insulin-induced hypoglycemia was similar in euthyroid and short- and longterm hyperthyroid animals (Fig. 1A). At 7 days,
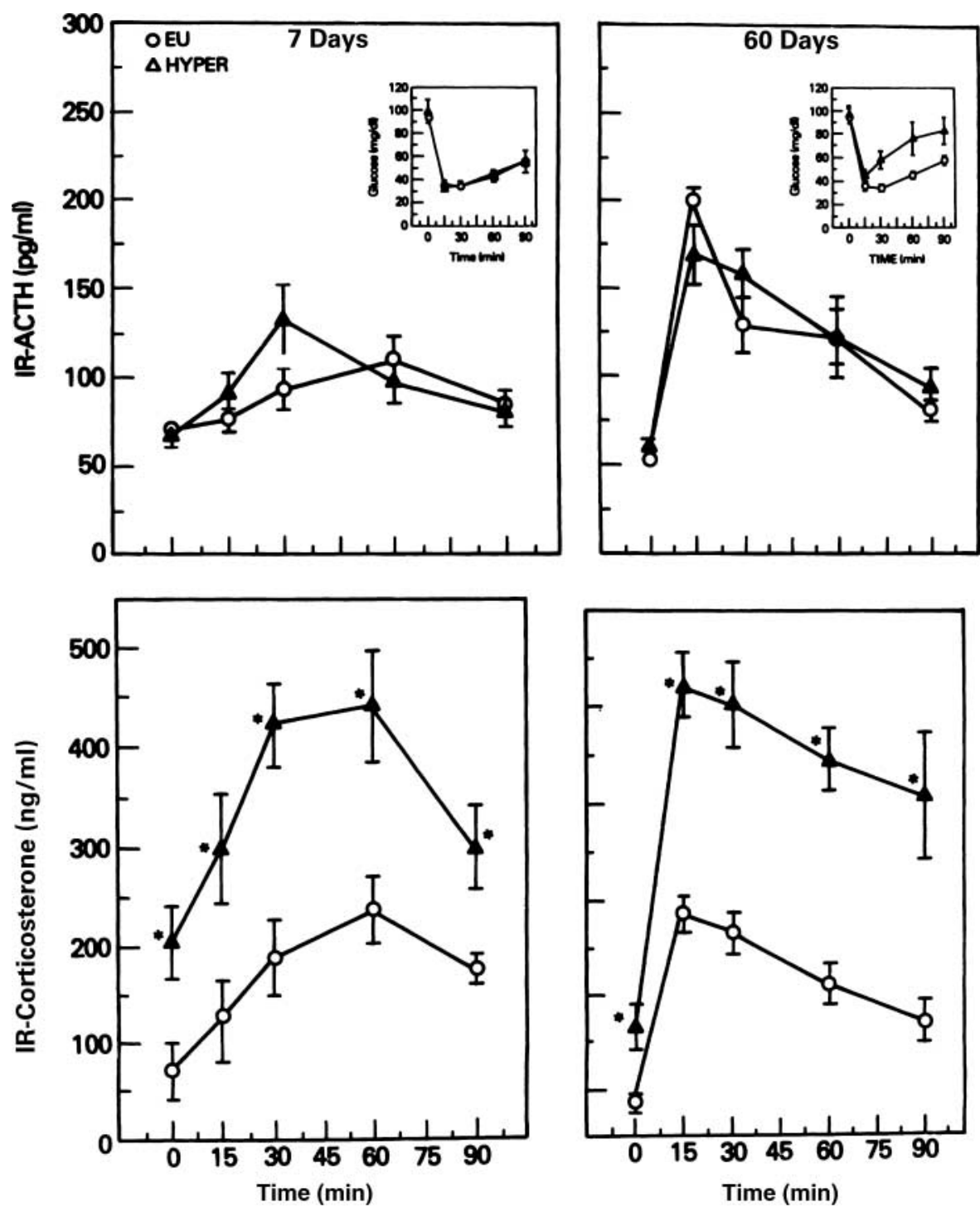

Figure 1 Responses of plasma IR-ACTH (panel A) and IR-corticosterone (panel B) to an i.v. bolus administration of $3.0 \mathrm{U} / \mathrm{kg}$ BW regular insulin in hyperthyroid or euthyroid freely moving, adult male rats 7 and 60 days after thyroidectomy plus $\mathrm{T} 4$ or sham-thyroidectomy plus placebo treatment. Inserts depict serum glucose levels. Values represent mean \pm S.E.M. of five or six different rats. ${ }^{\star} P<0.05$ vs the response elicited by insulin in euthyroid rats (by ANOVA followed by Fisher's PLSD). 

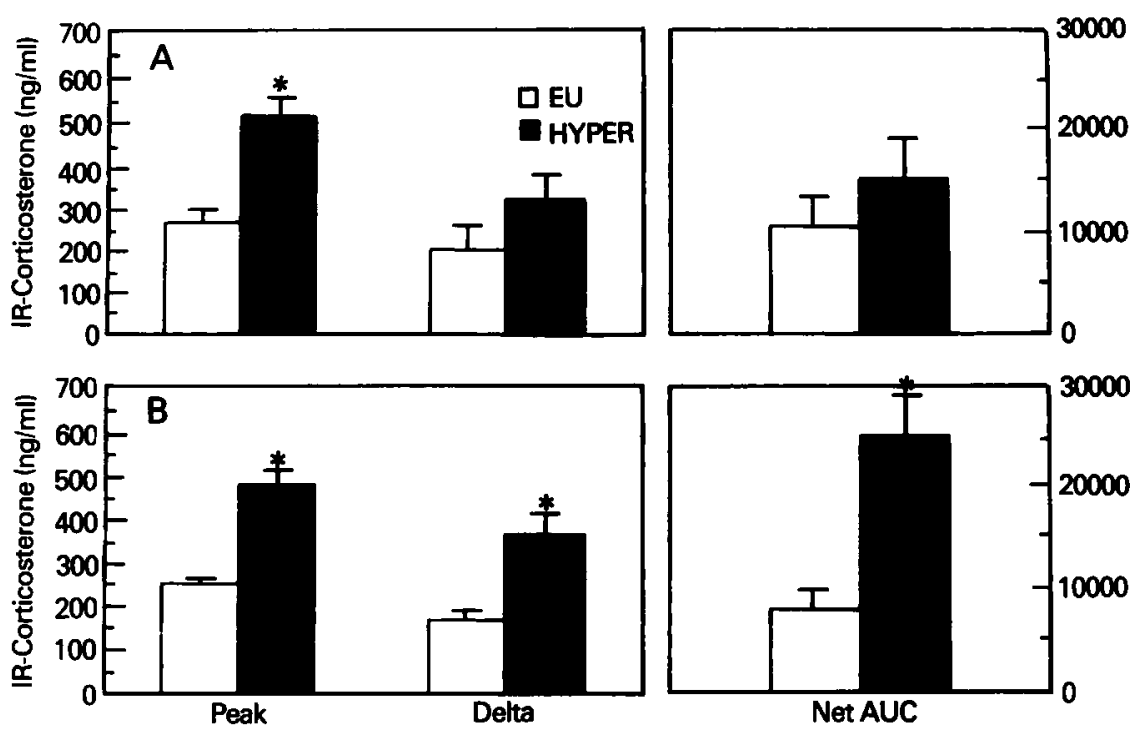

Figure 2 Peak, delta and net IR-corticosterone responses to an i.v. bolus administration of $3.0 \mathrm{U} / \mathrm{kg} \mathrm{BW}$ regular insulin in hyperthyroid or euthyroid freely moving, adult male rats $7(A)$ and 60 days $(B)$ after thyroidectomy plus T4 or sham-thyroidectomy plus placebo treatment. * $P<0.05$ vs the response elicited by insulin in euthyroid rats (by ANOVA followed by Fisher's PLSD). hyperthyroid rats showed an increased corticosterone response at each time point $(P<0.05$, by ANOVA followed by Fisher PLSD; Fig. 1), as well as a significant increase in the peak response point $(P<0.05$, by ANOVA followed by Fisher PLSD; Fig. 2A). Because of high basal corticosterone levels, the delta and net integrated corticosterone responses were similar to those in controls. By 60 days, hyperthyroid animals showed a significantly increased corticosterone response during hypoglycemic stress at each time point $(P<0.05$;
ANOVA followed by Fisher PLSD; Fig. 1B). Mean peak, delta and net integrated corticosterone response were also greater than controls after long-term hyperthyroidism $(P<0.05$, by ANOVA followed by Fisher PLSD; Fig. 2B).

Although plasma ACTH responses to IL- $1 \alpha$ administration were similar in long-term hyperthyroid rats and controls, the corticosterone response was significantly higher in hyperthyroid animals $(P<0.05$, by ANOVA followed by Fisher PLSD, Fig. 3). Although the peak
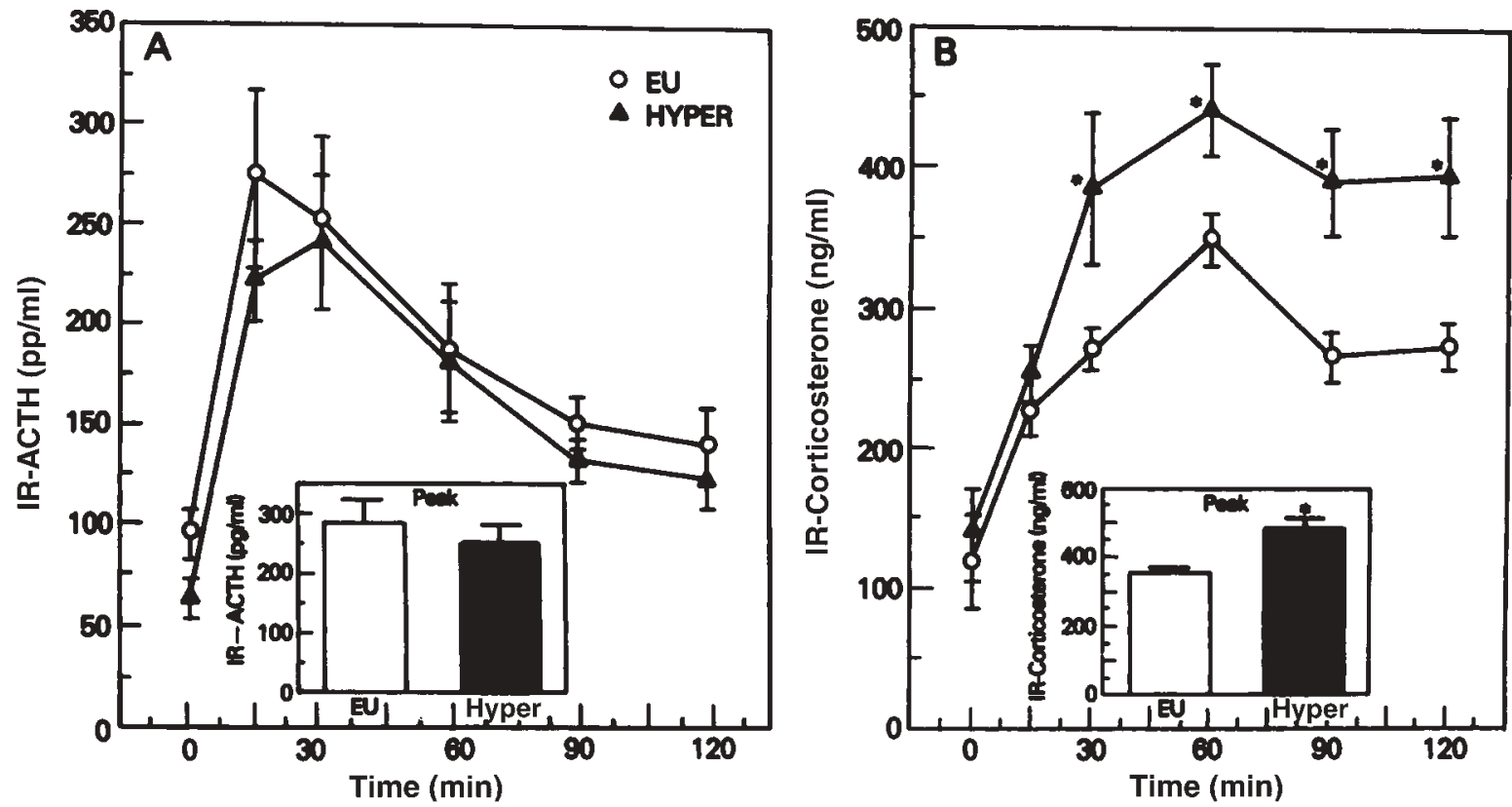

Figure 3 Responses of plasma IR-ACTH (A) and IR-corticosterone (B) to an i.v. bolus administration of $10 \mu \mathrm{g} / \mathrm{kg}$ BW recombinant IL$1 \alpha$ in hyperthyroid or euthyroid freely moving, adult male rats 60 days after thyroidectomy plus T4 or sham-thyroidectomy plus placebo treatment. Inserts depict peak plasma IR-ACTH and IR-corticosterone responses. Values represent means \pm S.E.M. of five or six different rats. ${ }^{\star} P<0.05$ vs the response elicited by IL- $1 \alpha$ in euthyroid rats (by ANOVA followed by Fisher's PLSD). 
IR-corticosterone response was significantly greater in the hyperthyroid animals $(P<0.05$, Fig. 3B insert), the delta and net integrated corticosterone responses were similar in long-term hyperthyroid rats and controls.

\section{Adrenal cortex}

Adrenal reserves, as indicated by the plasma corticosterone response to ACTH following dexamethasone suppression, were significantly elevated after short-term hyperthyroidism (Fig. 4A, $P<0.05$; ANOVA followed by Fisher PLSD). After short-term hyperthyroidism, the mean peak, delta and net-integrated corticosterone responses to exogenous ACTH administration were also significantly increased (Fig. 5, $P<0.05$; ANOVA followed by Fisher PLSD). In contrast, the corticosterone response at each time point was significantly lower in long-term hyperthyroidism (Fig. 4B, $P<0.05$; ANOVA followed by Fisher PLSD) compared with controls. No differences in the peak, delta and net-integrated corticosterone responses in long term hyperthyroidism were observed (Fig. 5).

\section{Thymus and adrenal weights}

Compared with euthyroid rats, there was a significant increase in the weights of the adrenals taken from both 7 and 60 day hyperthyroid animals $(P<0.05$; ANOVA followed by Fisher PLSD; Fig. 6A). The magnitude of the increase was the same in both short- and long-term treated animals. The weight of the thymus gland was significantly reduced in short-term hyperthyroid rats and was reduced to a much greater extent in long-term animals $(P<0.05$; ANOVA followed by Fisher PLSD; Fig. 6B).

\section{Discussion}

The data indicate that experimentally-induced hyperthyroidism is associated with sustained hypercortisolism, with both short- and long-term hyperthyroid rats showing significant elevations in total basal plasma corticosterone levels. These increases in basal corticosterone concentrations do not appear to be simply artifacts of hyperthyroidism-induced increases in basal CBG binding capacity, as the levels of corticosterone in the CSF are also increased in hyperthyroid rats. In this regard, as an ultrafiltrate of plasma, the CSF corticosterone concentration is thought to reflect the plasma free corticosterone levels. Significant increases in the corticosterone responses to the ACTH released during hypoglycemic stress and IL- $1 \alpha$ stimulation are also compatible with a hyperthyroid-induced hypercortisolism in the rat.

In addition to the significant increase in the CSF level of corticosterone, the postulate of hypercortisolism is further supported by our observation of a significant increase in the weight of the adrenals. The latter is almost never seen except in the context of enhanced pituitary stimulation of the adrenal cortex producing hypercortisolism $(8,17)$. Because a hyperthyroidmediated increase in the clearance of corticosterone or an increase in the CBG binding capacity in hyperthyroid rats would not be sufficient to influence free corticosterone levels in the absence of a change in the central or pituitary set-point for adrenocortical regulation, changes in clearance or in CBG binding
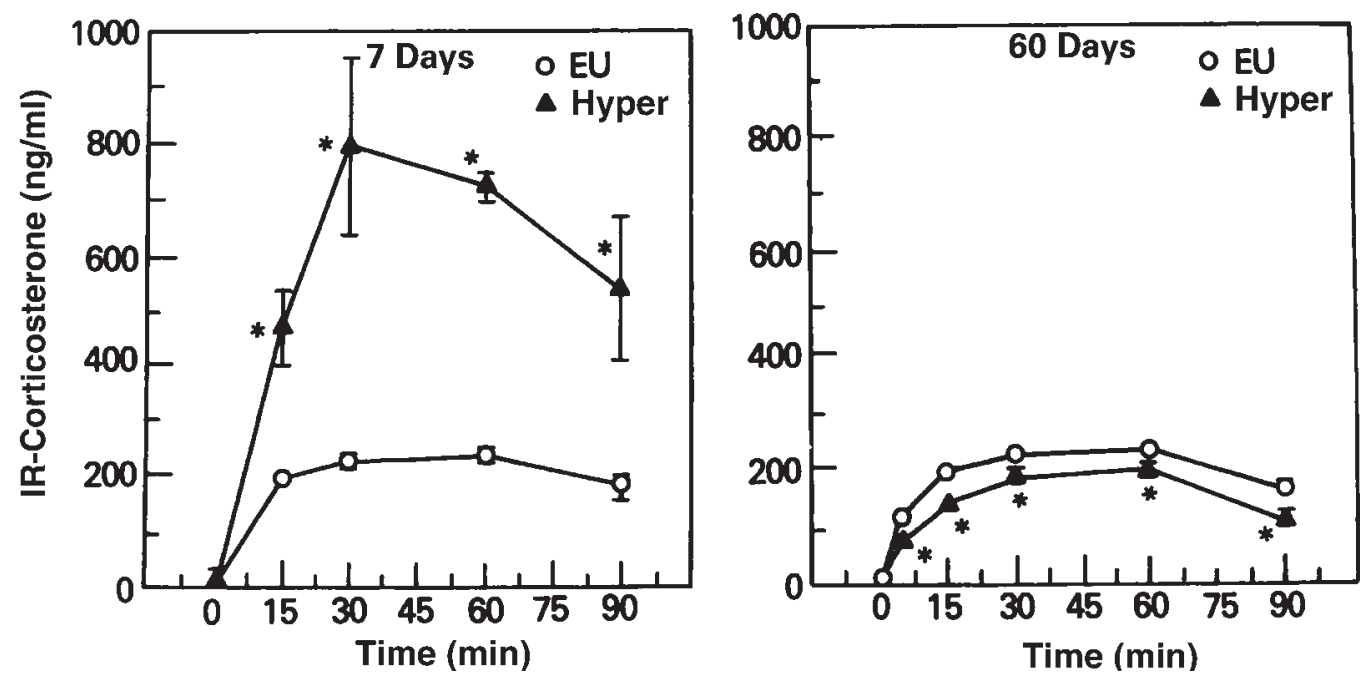

Figure 4 Responses of plasma IR-corticosterone to an i.v. bolus administration of $25 \mu \mathrm{g} / \mathrm{kg} \mathrm{BW}$ synthetic ACTH ${ }_{1-24}$ in hyperthyroid or euthyroid freely moving, adult male rats 7 and 60 days after thyroidectomy plus T4 or sham-thyroidectomy plus placebo treatment. Rats were injected i.m. $3 \mathrm{~h}$ previously with $200 \mu \mathrm{g}$ dexamethasone to inhibit endogenous ACTH secretion. Values represent mean \pm S.E.M. of five or six different rats. ${ }^{*} P<0.05$ vs the response elicited by ACTH in euthyroid rats (by ANOVA followed by Fisher's PLSD). 

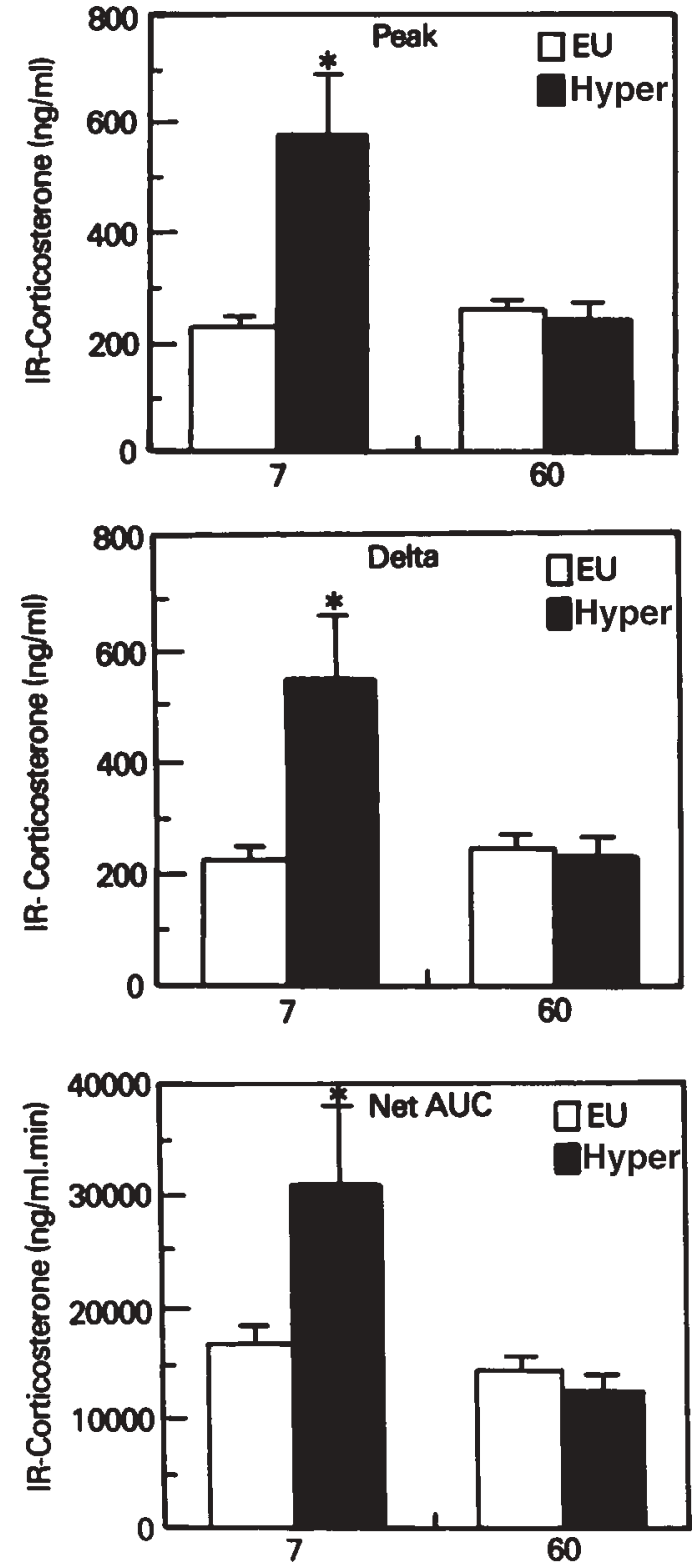

Figure 5 Peak $(A)$, delta $(B)$ and net area under the curve (AUC; C) plasma IR-corticosterone responses to an i.v. bolus administration of $25 \mu \mathrm{g} / \mathrm{kg} \mathrm{BW}$ synthetic $\mathrm{ACTH}_{1-24}$ in hyperthyroid or euthyroid freely moving, adult male rats 7 and 60 days after thyroidectomy plus T4 or sham-thyroidectomy plus placebo treatment. Rats were injected i.m. $3 \mathrm{~h}$ previously with $200 \mu \mathrm{g}$ dexamethasone to inhibit endogenous ACTH secretion. Values represent means \pm S.E.M. of five or six different rats. ${ }^{*} P<0.05$ vs the response elicited by ACTH in euthyroid rats (by ANOVA followed by Fisher's PLSD).

capacity cannot be invoked to account for the hypercortisolism seen in the hyperthyroid state.

Although we can not definitively document the site of the dysregulation of the HPA axis that is principally responsible for this hypercortisolism or the abnormalities of the hormonal responses to hypoglycemia and IL-1 $\alpha$ in hyperthyroidism, the weight of available data suggests that this hypercortisolism does not reflect a primary change in the functional integrity of the pituitary corticotroph cell. This is further supported by our earlier report of a reduced ACTH response to an acute bolus of oCRH in hyperthyroid rats (2). This mediates against the idea of a hyper-responsive corticotroph cell as a cause of the hypercortisolism in hyperthyroid rats. Rather, this blunted ACTH response seems most compatible with the enhanced restraint of the pituitary corticotroph cell by elevated levels of free corticosterone.

We cannot rule out either a central or a primary adrenal locus as a principal site for hyperthyroid-mediated hypercortisolism. However, symmetrical enlargement of the adrenals in the context of hypercortisolism has almost always been associated with physiological contexts in which there has been hyperstimulation of the adrenal cortex with ACTH, rather than with the context of a primary adrenal process $(8,18,19)$. In light of our previous oCRH stimulation studies, this would suggest a central locus for the hyperthyroid-mediated hypercortisolism.

The present study has shown that adrenocortical reserve, as assessed by direct stimulation with a low dose ACTH following dexamethasone pretreatment, is attenuated in long-term hyperthyroidism. Previous studies have examined the effects of altered thyroid function on the secretion and metabolism of adrenocortical hormones. In thyrotoxic states the degradation of cortisol is accelerated, but its rate of production is also increased $(5,10,12,13)$. These observations indicate that a degree of adrenal cortical hypersensitivity would be sustained in hyperthyroidism as a response to increased need. We have shown in a previous report that patients with severe thyrotoxicosis, cortisol secretion in response to low-dose ACTH stimulation, following dexamethasone suppression, was lower in the hyperthyroid, than in the euthyroid state (1). These findings suggest that thyrotoxicosis in humans is associated with a subtle impairment of adrenocortical reserve. This is compatible with our present findings in long-term hyperthyroid animals, in which despite the continuing hyperactivity of the HPA axis, the corticosterone response to direct stimulation with adrenocorticotropin following dexamethasone suppression, was lower than normal. These findings suggest that in long-term hyperthyroidism adrenal functional reserve might be compromised.

The findings that experimentally-induced hyperthyroidism is associated with diminished adrenocortical reserves in a time-dependent fashion has not only theoretical implications, but clinical implications as well. Thyroid storm has been associated with subclinical hypoadrenocorticism (20). Also in thyroid storm, cortisol levels, while within the normal range, have been found to be lower than generally seen during periods of stress, suggesting relative adrenal insufficiency (21). Indeed, in thyrotoxic crisis, treatment 

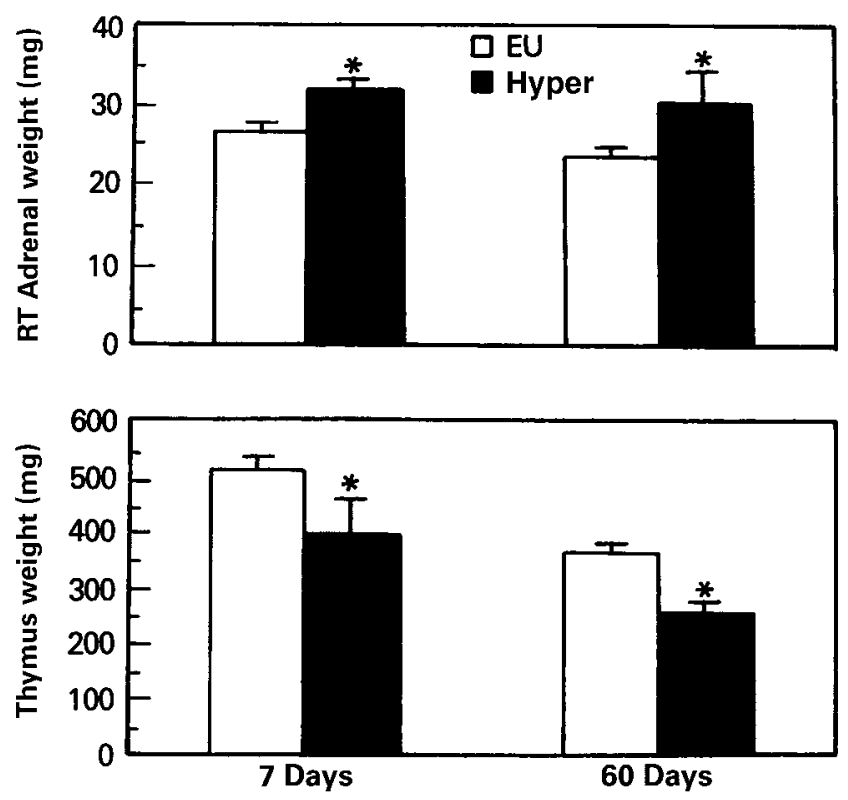

Figure 6 Effects of short- and long-duration hyperthyroidism on thymus (A) and adrenal weights (B). Values represent means \pm S.E.M. Short- and long-duration hyperthyroidism were associated with increased adrenal weights. In contrast, short- and long-duration hyperthyroidism resulted in a significant reduction in thymus weights. ${ }^{*} P<0.05$ vs tissue weight in euthyroid rats (by Student's $t$-test). with hydrocortisone is recommended because of the likelihood of associated adrenal insufficiency $(22,23)$.

Despite the available clinical and experimental evidence, there is disagreement as to whether adrenal functional reserve is adequate in clinical hyperthyroidism. In this regard, sensitive tests for assessing adrenal functional reserve become of significant importance. In recent years, stimulation with low dose ACTH has been used as a sensitive test to detect subtle changes in adrenal function, which would be missed if the standard ACTH test is used (24-26). The low dose ACTH test following dexamethasone suppresion of endogenous ACTH, is a sensitive method to assess adrenocortical reserve in vivo.

The mechanism for the attenuated adrenal reserve in long-term hyperthyroidism is not clear considering that many factors may influence serum glucocorticoid concentrations. It has been demonstrated that hyperthyroidism increases the secretion rate of cortisol, influences the rate of cortisol degradation and affects its metabolism qualitatively (14). Previous experimental studies have also shown that adrenal steroidogenesis is impaired by pharmacological doses of T4 (19). It appears that the duration and severity of the hyperthyroid state may be important factors determining the level of the functional capacity of the adrenals and the HPA axis. It may be that, in sustained hyperthyroidism, the adrenals secrete at their maximal rate to keep up with the increased metabolic degradation of cortisol and thus, their reserve in response to further stimulation with ACTH is diminished. This burden may be more severe in thyroid storm culminating in a relative adrenal insufficiency.
The possibility that the findings are the result of increased clearance of ACTH and cortisol need further consideration in the interpretation of the data. We addressed this question in a previous study, which showed that the clearance of $\mathrm{ACTH}_{1-24}$ from plasma was not significantly affected in experimentally induced hyperthyroidism (2). If the findings were simply the result of the increased clearance of ACTH and/or corticosterone, it would be expected that not only the magnitude of the glucocorticoid response in the hyperthyroid state would be smaller, but also the duration of the response would be shorter, which was not evident in our data. In addition, the prior suppression of endogenous ACTH and cortisol by dexamethasone pretreatment allowed only the de novo secretion of cortisol in response to acute ACTH to be measured and this would mainly reflect adrenal reserves.

In summary, our data indicate that experimentallyinduced hyperthyroidism is associated with significant alterations in hypothalamic-pituitary-adrenal axis function that are contingent upon the duration of the alterations in thyroid function. Significant elevations in basal plasma corticosterone levels, and significant increases in the corticosterone response to the ACTH released during hypoglycemic stress and IL- $1 \alpha$ stimulation, suggest hyperthyroid-induced hypercortisolism. Despite the sustained hyperactivity of the HPA axis, long-term experimentally-induced hyperthyroidism was associated with attenuated adrenal corticosterone responses to ACTH stimulation following prior suppression with dexamethasone. These findings indicate that sustained hyperthyroidism may be associated with diminished adrenocortical reserve. 


\section{Acknowledgements}

Material for the rat TSH RIA were provided by the National Hormone and Pituitary Program.

\section{References}

1 Tsatsoulis A, Johnson EO, Kalogera CH, Seferiadis K \& Tsolas O. The effect of thyrotoxicosis on adrenocortical reserve. European Journal of Endocrinology 200042 231-235.

2 Kamilaris TC, DeBold CR, Johnson EO, Mamalaki E, Listwak SJ, Calogero AE, Kalogeras KT, Gold PW \& Orth DN. Effects of short and long duration hypothyroidism and hyperthyroidism on the plasma adrenocorticotropin and corticosterone responses to ovine corticotropin-releasing hormone in rats. Endocrinology $19911282567-2576$.

3 Harvard CWH, Saldanha VF, Bird R \& Gardner R. Adrenal function in hyperthyroidism. British Medical Journal 19701 $337-339$

4 Giustina G, Reschini E, Valentini F \& Cantalamess L. Growth hormone and cortisol responses to insulin-induced hypoglycemia in thyrotoxicosis. Journal of Clinical Endocrinology and Metabolism $197132571-574$.

5 Levin ME \& Daughaday WH. The influence of the thyroid on adrenocortical function. Journal of Clinical Endocrinology and Metabolism 195515 1499-1511.

6 Peterson RE. The influence of the thyroid on adrenal cortical function. Journal of Clinical Investigation 195837 736-743.

7 Sanchez-Franco F, Fernandez L, Fernandez G \& Cacicedo L. Thyroid hormone action on ACTH secretion. Hormone Metabolism Research 198921 550-552.

8 Steinetz BG \& Beach VL. Some influences of thyroid on the pituitary-adrenal axis. Endocrinology 196372 45-72.

9 D'Agostino J \& Henning SJ. Role of thyroxine in coordinate control of corticosterone and $\mathrm{CBG}$ in postnatal development. American Journal of Physiology 1982242 E33-E39.

10 Gordon GG \& Southren AL. Thyroid hormone effects on steroid hormone metabolism. Bulletin of the New York Academy of Medicine $197753241-259$.

11 Kamilaris TC, DeBold CR, Pavlou SN, Island DP, Hoursanidis A \& Orth DN. Effect of altered thyroid hormone levels on hypothalamic-pituitary-adrenal function. Journal of Clinical Endocrinology and Metabolism 198765 994-999.

12 Peterson RE \& Wuygaarden JB. The miscible pool and turnover rate of hydrocortisone in man. Journal of Clinical Investigation $195638552-556$

13 McGuire JS \& Tomkins GM. The effects of thyroxine administration on the enzymatic reduction of delta 3-ketosteroids. Journa of Biological Chemistry 1959234 729-734.
14 Gallagher TF, Hellman L, Finkelstein J, Yoshida K, Weitzman ED, Roffward HD \& Fukushima DK. Hyperthyroidism and cortisol secretion in man. Journal of Clinical Endocrinology and Metabolism $197234919-927$

15 Calogero AE, Bagdy G, Szemeredi K, Tartaglia ME, Gold PW \& Chrousos GP. Mechanisms of serotonin receptor agonist-induced activation of the hypothalamic-pituitary-adrenal axis in the rat. Endocrinology $1990 \mathbf{1 2 6} 1888-1894$.

16 Calogero AE, Kamilaris TC, Bernardini R, Johnson EO, Chrousos GP \& Gold PW. Effects of peripheral benzodiazepine receptor ligands on hypothalmic-pituitary-adrenal axis function in the rat. Journal of Pharmacology and Experimental Therapeutics $1990253729-737$.

17 Dallman MF, Akana SK, Cascio CS, Darlington DN, Jacobson L \& Levin N. Regulation of ACTH secretion: Variations on a theme of B. Recent Progress in Hormone Research 198743 113-167.

18 Moore NA \& Callas G. The effects of hyperthyroidism on the fine structure of the zona fasciculata of rat adrenal cortex. Anatomical Records $1972 \mathbf{1 7 4} 451-459$.

19 Boler RK \& Moore NA. Depression of adrenocortical function by pharmacologic doses of thyroxine in intact and unilaterally adrenalectomized rats. Hormone Research $198216209-218$.

20 Kawaguch M, Iwata S, Kamiya Y, Hayakawa F, Fujii T, Ito J, Sakuma N \& Fujinami T. Thyroid storm associated with probable subclinical hypoadrenocortiscism in an elderly woman. Internal Medicine 199231 1236-1238.

21 Mazzaferri MEZ \& Skillman TG. Thyroid storm. Archives of Internal Medicine $1969124684-690$.

22 Ingbar SH. Management of emergencies. IX. Thyroid storm. New England Journal of Medicine 1966274 1252-1254.

23 Burch HB \& Wartofsky L. Life-threatening thyrotoxicosis. Endocrinology and Metabolism Clinics of North America 199322 $263-277$.

24 Dickstein G, Schechner C, Nicholson WE, Rosner I, Shen-Orr Z Adawi F \& Lahan M. Adrenocorticotropin stimulation test: effects of basal cortisol level, time of day and suggested new sensitive low dose test. Journal of Clinical Endocrinology and Metabolism 199172 $773-779$.

25 Crowley S, Hindmarsch PC, Holownia P, Honour JW \& Brook CGD. The use of low doses of ACTH in the investigation of adrenal function in man. Journal of Endocrinology $1991130425-479$.

26 Dickstein G, Spigel D, Frad E \& Shechner C. One microgram is the lowest ACTH dose to cause maximal cortisol response. There is no diurnal variation of cortisol response to submaximal ACTH stimulation. European Journal of Endocrinology 1997137 172-175.

Received 17 November 2004

Accepted 9 March 2005 